\title{
AVALIAÇÃO DA ATIVIDADE DA LIPOXIGENASE EM LINHAGENS DE SOJA (')
}

\author{
HAIKO ENOK SAWAZAKI $(2)$, JOĀO PAULO FEIJĀO TEIXEIRA $(2,4)$ \\ Q MANOEL ALBINO COELHO DE MIRANDA $(3,4)$
}

\begin{abstract}
RESUMO
Com o fim de verificar os melhores métodos para identificação de mutantes de soja para a enzima lipoxigenase e a possivel correlação entre atividade enzimática e teor de ácido linolênico, foram analisadas sementes do cultivar IAC-8 e das linhagens A-5 (de baixo teor de ácido linolênico), PI 408251 (mutante menos $L_{1}$ ), PI 86023 (mutante menos $L_{2}$ ), Tohoko $n^{2} 74$ (mutante menos $L_{3}$ ), e as da geração $F_{2}$ do cruzamento do 'IAC-8' com as linhagens mutantes (menos $L_{1}$, menos $L_{2}$ e menos $L_{3}$ ), quanto aos teores de ácido linolênico, atividade de lipoxigenase e ocorrência de genótipos com ausência de isoenzima de lipoxigenase. A quantificação da atividade enzimática foi realizada por método colorimétrico e a detecção das isoenzimas de lipoxigenase, por focalização isoelétrica. O teor de óleo foi determinado gravimetricamente e o de ácidos graxos, por cromatografia em fase gasosa de seus ésteres metilicos. A determinaçāo da atividade enzimática forneceu boa indicação para identificação final das isoenzimas por focalização isoelétrica, permitindo o emprego desse método para a escolha de linhagens em programa de melhoramento genético visando a maior estabilidade do óleo de soja. Para o material estudado, não se encontrou correlação entre atividade enzimática e teor de ácido linolênico.
\end{abstract}

Termos de indexaçāo: soja, Glycine max (L) (Merrill), lipoxigenase, ácido linolênico.

(1) Recebido para publicação em

(2) Seção de Fitoquímica, Instituto Agronômico (IAC), Caixa Postal 28, 13001 Campinas (SP).

(3) Seção de Leguminosas, IAC.

(4) Com bolsa de suplementação do CNPq. 


\section{INTRODUÇÃO}

Fatores antinutricionais da soja, como inibidores de tripsina e quimotripsina, fatores bociogênicos e hemaglutininas, são inativados por tratamento térmico em umidade adequada, de forma a ser efetivo e a não diminuir muito a solubilidade da proteína. A desnaturação térmica influi nas propriedades físicas de gelificação, texturização ou fibrilação da proteína. $O$ tratarnento térmico excessivo empobrece o valor nutritivo, diminuindo os teores de aminoácidos sulfurados sensíveis ao calor e a disponibilidade da lisina: com a hidrólise dos carboidratos, ocorre o aumento do nivel dos acúcares redutores, que reagem com os grupos $\alpha$-amino da lisina na reaçāo de Maillard.

A ativação da enzima lipoxigenase, pelo aumento da umidade necessária para a efetividade do tratamento térmico, origina a oxidação dos ácidos graxos poliinsaturados com o sistema pentadieno, principalmente o ácido linolênico, desenvolvendo sabor e odor desagradáveis (MUSTAKAS et al., 1969, e SESSA et al., 1969).

MATTICK \& HAND (1969) isolaram 80 compostos voláteis que acharam ser o resultado da atividade da lipoxigenase, identificando 40 , sendo grande a concentração de $n$-hexanal e $n$-hexanol. Identificaram entre estes o principal causador do sabor desagradável, semelhante ao "green-beany", que não está presente na soja crua, mas se desenvolve imediatamente após maceração do grāo, devido à autoxidação do óleo de soja.

Métodos utilizados para a melhoria das caracteristicas organolépticas, como o tratamento térmico, extração com solventes orgânicos, utilização, segundo CHIBA et al. (1979), da enzima aldeído-desidrogenase (que catalisa a conversão irreversivel dos aldeídos em seus ácidos correspondentes), têm sido estudados.

Em relação ao óleo, os "off flavors" podem ser removidos por processos de refinação, mas os componentes de degradação do odor e sabor permanecem, causando o desenvolvimento do gosto desagradável, sendo necessária a hidrogenação para aumentar a estabilidade do óleo.

A literatura relata a existência de isoenzimas de lipoxigenase em soja, por gel eletroforese de disco, com coloração específica das bandas enzimaticamente ativas (GUSS et al., 1967, 1968).

CHRISTOPHER et at. (1970) isotaram uma isoenzima, distinta da lipoxigenase tipo 1 , anteriormente conhecida como a dos ácidos graxos. Devido à especificidade de substrato sobre uma faixa de $\mathrm{pH}$, relataram ser a enzima tipo 2 a responsável pela lipoxigenase dos triglicerídeos, corroborando $\mathrm{KOCH}$ et al. (1958).

CHRISTOPHER et al. (1972) isolaram uma terceira isoenzima da lipoxigenase de soja, distinta das lipoxigenases 1 e 2 . Relataram os pontos isoelétricos de 5,68 para a $1 ; 6,25$ para a 2 e 6,15 para a 3 . As lipoxigenases 1 e 2 apresenta- 
ram pH ótimo, respectivamente 9,5 e 6,5. A tipo 3 mostrou atividade na faixa de $\mathrm{pH} \mathrm{4,5}$ a aproximadamente 9,0 .

KITAMURA (1984) identificou três linhagens mutantes de soja que não apresentaram as bandas das isoenzimas da lipoxigenase, a menos $L_{1}$, a menos $L_{2}$ e a menos $L_{3}$, por método gel eletroforese com dodecilsulfato de sódio (SDS).

VERHUE \& FRANCKE (1972) e FUNK et al. (1985), através de focalização isoelétrica e coloração específica das bandas com atividade enzimática, resolveram as duas classes principais de isoenzimas (tipos 1 e 2 ).

Os alelos nulos $I x_{1}, \mid x_{3}$ e $\mid x_{2}$ foram identificados para as proteinas $L_{1}, L_{s}$ e $L_{2}$ por HILDEBRAND \& HYMOWTZ (1982), KITAMURA et al. (1983) e DAVIES \& NIELSEN (1986). Esses autores identificaram os genótipos duplos recessivos $\left|x_{1}\right| x_{1}\left|x_{3}\right| x_{3}$ e $\left|x_{2}\right| x_{2}\left|x_{3}\right| x_{3}$ e a ligação dos genes das proteinas $L_{1}$ e $L_{2}$.

Sendo possivel a diminuição da atividade da lipoxigenase pela incorporação dos genes mutantes recessivos, procurou-se pesquisar, neste trabalho, os melhores métodos para a identificação do nivel e tipo de atividade enzimática, assim como sua correlação yuanto aos teores do ácido linolênico.

\section{MATERIAL E MÉTODOS}

Utilizaram-se as sementes de soja Glycine max (L.) Merrill, dos cultivares IAC-8 (comercial) e das linhagens A-5 (de lllinois, de baixo teor de ácido linolênico, HAMMOND \& FEHR, 1983), PI 408251 (mutante menos $L_{1}$ ), PI 86023 (mutante menos $L_{2}$ ) e Tohoko $n^{0} 74$ (mutante menos $L_{3}$ ), da colheita de setembro de 1985, e as sementes da geração $F_{2}$ do cruzamento do 'IAC-8' com os mutantes menos $L_{1}$, menos $L_{2}$ e menos $L_{3}$, da colheita de março de 1986.

O substrato empregado foi extraído do óleo de sementes de Manihot catingae Ule contendo $85 \%$ de ácido linoléico.

Para diferenciação dos cultivares realizaram-se as seguintes análises quantitativas e qualitativas: (a) extração de lipídios por hexano em Soxhlet (TRIEBOLD \& AURAND, 1963); (b) ácidos graxos, esterificados de acordo com HARTMAN \& LAGO (1973), por cromatografia em fase gasosa, utilizando coluna de vidro $6^{\prime} \times 1 / 8^{\prime \prime}$ com fase líquida DEGS $10 \%$, suporte Chromosorb WAW, $60 / 80$ mesh, sob temperatura de $180^{\circ} \mathrm{C}$; (c) lipoxigenase, pelo método do tiocianato de $\mathrm{KOCH}$ et al. (1958) modificado, que mede a formação de hidroperóxidos; (d) proteina solúvel, pelo método de LOWRY et al. (1951); (e) gel eletroforese com SDS, de KITAMURA (1984); (f) focalizaçāo isoelétrica com coloração enzimática (VERHUE \& FRANCKE, 1972, e FUNK et al., 1985).

\subsection{Extração de lipoxigenase}

Moeram-se os grãos até obtenção de farinha com granulometria de 20 malhas, que foi guardada a $4^{\circ} \mathrm{C}$. Na farinha desengordurada pelo método de 
Soxhlet, efetuou-se a extração de lipoxigenase pela homogeneização em tampão tris- $\mathrm{HCl} \mathrm{pH} 7,0,0,05 \mathrm{M}$, com dez minutos de ultra-som por três vezes e centrifugação a $10.000 \mathrm{rpm}$ por dez minutos a $4^{\circ} \mathrm{C}$. Utilizou-se a relação $1: 10$ ou 1:100 (grama de amostra/mililitro de tampão) para a análise qualitativa ou quantitativa.

Manteve-se o extrato enzimático a $4^{\circ} \mathrm{C}$, utilizando-se pipetas capilares para evitar aderência da enzima às paredes de vidro dos recipientes (ALLEN, 1968).

\subsection{Modificação do método do tiocianato}

a) Reação enzimática

Pouco antes de se efetuar a reação enzimática, diluiu-se o extrato enzimático de 20 a 40 vezes, pois, uma vez diluída, a atividade é mais facilmente perdida (DILLARD et al., 1961). A solução estoque do substrato foi constituída de $0,01 \mathrm{~g}$ de ácido linoléico. $\mathrm{ml}^{-1}$ de etanol.

Efetuou-se a reação enzimática, misturando-se, em tubos de ensaio, $0,2 \mathrm{ml}$ de extrato enzimático diluído com $0,2 \mathrm{ml}$ de solução de substrato diluído (1:10) em tampão tris- $\mathrm{HCl} 0,2 \mathrm{M}, \mathrm{pH} 8,4$ ou 7,0 , com ou sem $0,05 \mathrm{mM} \mathrm{Ca}^{2+}$. A reação foi interrompida aos cinco minutos pela adição de $3 \mathrm{ml}$ de álcool etílico.

b) Reação de coloração dos hidroperóxidos

Adicionou-se ao tubo com reação enzimática interrompida $0,1 \mathrm{ml} \mathrm{HCl}$ $20 \%(\mathrm{v} / \mathrm{v})$ e $0,05 \mathrm{ml}$ de solução $\mathrm{Fe}^{2+} 0,1 \%$. Agitou-se e, após 30 segundos, acrescentou-se $0,1 \mathrm{ml}$ de tiocianato de amônio $10 \%$. Decorridos $5-15$ minutos fez-se a leitura, a $480 \mathrm{~nm}$. A solução estoque de $\mathrm{Fe}^{2+}$ consistiu em $5 \%$ de sulfato ferroso amoniacal em $\mathrm{HCl} 3 \%(\mathrm{v} / \mathrm{v})$.

\subsection{Determinação qualitativa}

Para a determinação do perfil eletroforético das isoenzimas da lipoxigenase $L_{1}, L_{2}$ e $L_{3}$, utilizou-se o método SDS-PAGE de KITAMURA (1984) e o de focalização isoelétrica como se segue. A extração foi realizada com tampão $\mathrm{pH}$ $7,0,0,05 \mathrm{M}$ (1:10), com extração dos pectatos de cálcio, de acordo com FUNK et al. (1985). O gel de $13 \times 11 \times 0,1 \mathrm{~cm}$, consistiu em $7 \%$ de acrilamida, $0,2 \%$ de $\mathrm{N}-\mathrm{N}$-metileno-bis-acrilamida, $0,05 \%$ tetrametiletilenodiamina, $1 \%$ de anfolitos $(0,53 \%$ de $\mathrm{pH} 4,0-6,5,0,27 \%$ de $\mathrm{pH} 4,5-9,0$ e $0,20 \%$ de $\mathrm{pH} 3,0-10,0), 0,0003 \%$ de riboflavina e $0,0033 \%$ de persulfato de amônia. Os compartimentos do ânodo (inferior) e cátodo (superior) foram preenchidos com $\mathrm{H}_{3} \mathrm{PO}_{4} 0,1 \mathrm{M}$ e $\mathrm{NaOH} 0,2 \mathrm{M}$. A coloraçăo das bandas para lipoxigenase, de acordo com VERHUE \& FRANCKE (1972), foi feita pela imersão do gel em meio contendo $0,1 \%(\mathrm{p} / \mathrm{v}) \sigma$-dianisidina, $0,1 \%(\mathrm{p} / \mathrm{v})$ ácido linoléico e $10 \%(\mathrm{v} / \mathrm{v})$ etanol em tampão $\mathrm{NaPi} \mathrm{pH} 7,00,1 \mathrm{M}$. Para prọteína, pela imersão em meio contendo $0,05 \%$ de Coomassie blue e $0,1 \%$ de $\mathrm{CuSO}_{4}$ em ácido acético-etanol- $\mathrm{H}_{2} \mathrm{O}$ (RIGHETTI \& DRYSDALE, 1974). 


\section{RESULTADOS E DISCUSSÃO}

Inicialmente, determinou-se a atividade da lipoxigenase da amostra IAC-8 pelo método do tiocianato, verificando-se que para ocorrer quase toda a reação enzimática do extrato de $1000 \mu \mathrm{g}(1: 10)$ de amostra, são necessários 4 minutos $\mathrm{e}$ $0,713 \mu \mathrm{mol}$ do substrato ácido linoléico, em um volume de $0,4 \mathrm{ml}$.

Realizadas as reações dos extratos correspondentes a $1.000 \mathrm{\mu g}$ das amostras IAC-8, menos $L_{1}$, menos $L_{2}$ e menos $L_{3}$, por 5 minutos, em volume de $0,4 \mathrm{ml}$, observou-se a atividade máxima quando a concentração do substrato variou de 0,178 a 0,267 mM. O 'IAC-8' teve maior atividade enzimática, requerendo mais substrato, enquanto a menos $L_{1}$, de menor atividade, sofreu maior inibiçāo com o aumento do substrato (Figura 1). Os valores de $K_{M}$, considerando-se apenas as concentraçōes iniciais do substrato, foram de 0,024, 0,044, 0,032 e $0,030 \mathrm{mM}$, indicando que o 'IAC-8' teve reação mais rápida, seguido pelo menos $L_{3}$, menos $L_{2}$ e menos $L_{1}$.

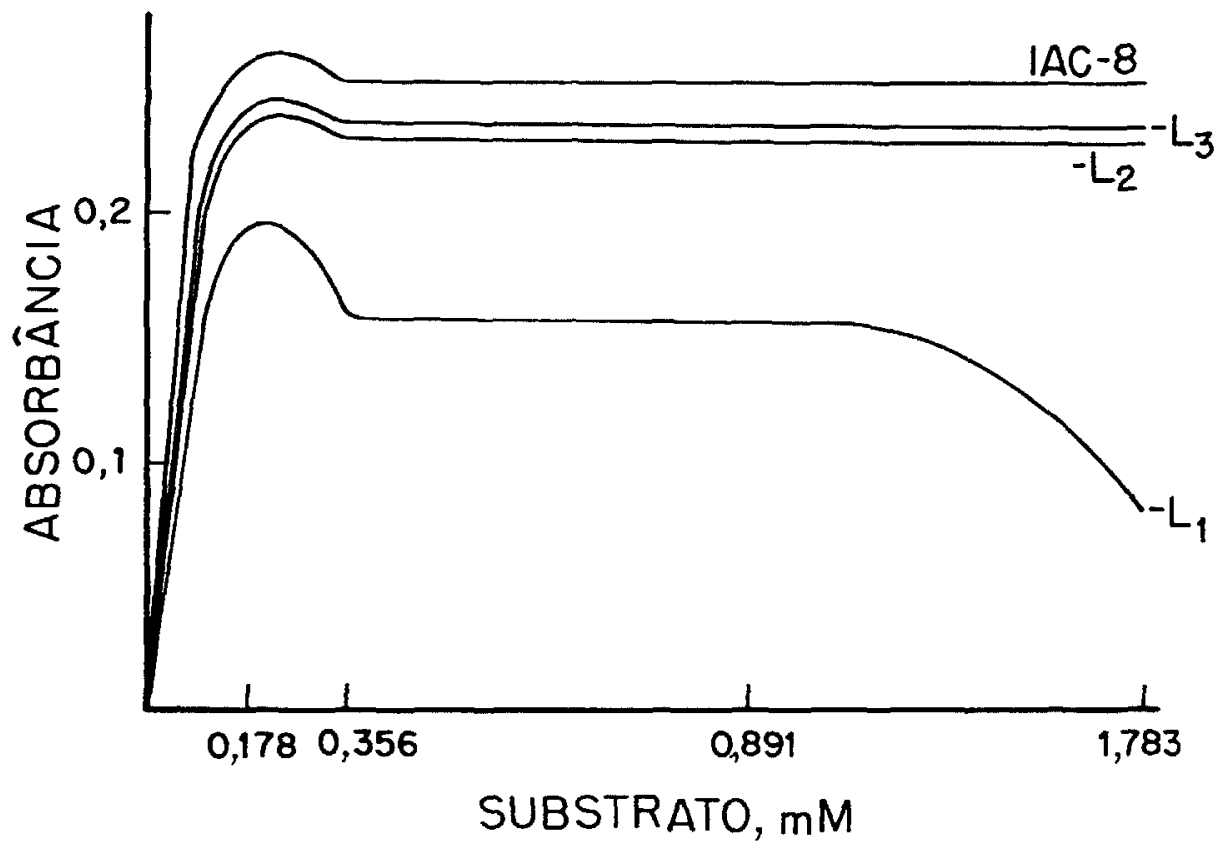

FIGURA 1. Relação entre velocidade de reação de lipoxigenase e concentração de substrato. 
Em vista de os resultados das reações dos extratos enzimáticos de $1.000 \mathrm{ug}$ de amostra com $1,783 \mathrm{mM}$ de substrato quase não determinarem diferenças entre os cultivares, procurou-se determinar a quantidade de amostra necessária para se obter a caracterização das amostras, cujos resultados em atividade específica estão no quadro 1.

A leitura da atividade enzimática em absorbância, correlacionada com o conteúdo protéico do extrato enzimático, obtido de acordo com LOWRY et al. (1951), é apresentada como a atividade específica (AE):

$$
A E=\frac{100 \times \text { absorbância enzima }}{\text { absorbância protéica }}
$$

Verificou-se que, a partir do extrato de $60 \mu \mathrm{g}$ de amostra, ocorreu maior diferença entre o cultivar IAC-8 e as linhagens.

Nesse caso, a extração foi feita na razão de 1:100.

Pelo quadro 2, verifica-se que a quantidade de ácidos graxos insaturados, seja com duas (linoléico), seja com três (linolênico) ligações duplas, não pareceu correlacionar negativamente com a atividade enzimática, ou seja, para menor atividade não ocorreu maior quantidade do ácido linolênico. A linhagem A-5 revelou baixo índice de ácido linolênico, embora a atividade enzimática não fosse muito inferior à do IAC-8.

O quadro 3 apresenta os resultados, em absorbância, das análises de lipoxigenases no pH 8,4 e no pH 7,0 com e sem $\mathrm{Ca}^{2+}$, das amostras da geração $F_{2}$ do cruzamento 'IAC-8' $X$ mutante identificadas como mutantes por focalização isoelétrica.

QUADRO 1. Atividade específica ( $A E$ ) do extrato enzimático (ou de lipoxigenase) de quantidades variáveis de amostras na reação com $1,783 \mathrm{mM}$ de substrato, $\mathrm{pH} 8,40,05 \mathrm{mM}$ $\mathrm{Ca}^{2+}$, volume total $0,4 \mathrm{ml}$

\begin{tabular}{lrrrrrr}
\hline \multirow{2}{*}{ Cultivar } & \multicolumn{7}{c}{ Amostra $(\mu g)$} \\
\cline { 2 - 6 } & 400 & 250 & 100 & 80 & 60 & 40 \\
\hline PI 408251 $\left(-L_{1}\right)$ & 22 & 9 & 3 & 18 & 12 & 10 \\
PI 86023 $\left(-L_{2}\right)$ & 41 & 109 & 69 & 126 & 188 & 126 \\
Tohoko no $74\left(-L_{3}\right)$ & 41 & 109 & 69 & 126 & 189 & 126 \\
A-5 & 39 & 102 & 112 & 206 & 225 & 170 \\
IAC-8 & 44 & 111 & 114 & 207 & 259 & 258 \\
\hline
\end{tabular}


QUADRO 2. Variação nos teores de óleo e de ácidos graxos de grãos de soja

\begin{tabular}{lcccccc}
\hline \multirow{2}{*}{ Cultivar } & Óleo & \multicolumn{5}{c}{ Ácidos graxos } \\
\cline { 3 - 7 } & & Palmítico & Esteárico & Oléico & Linoléico & Linolênico \\
\hline & $\%$ & & & $\%$ & & \\
Pl 408251 $\left(-L_{1}\right)$ & 17,56 & 13,71 & 1,42 & 17,93 & 58,67 & 8,26 \\
PI 86023 $\left(-L_{2}\right)$ & 17,86 & 16,64 & 1,35 & 25,83 & 49,39 & 6,80 \\
Tohoko $n^{\circ} 74\left(-L_{3}\right)$ & 14,30 & 13,53 & 1,20 & 18,68 & 57,92 & 8,66 \\
A-5 & 20,69 & 12,85 & 1,31 & 44,55 & 38,89 & 2,40 \\
IAC-8 & 20,20 & 14,18 & 1,47 & 23,63 & 52,43 & 8,29 \\
\hline
\end{tabular}

QUADRO 3. Niveis da lipoxigenase (em absorbância)

\begin{tabular}{|c|c|c|c|}
\hline \multirow{2}{*}{ Cultivar } & \multirow{2}{*}{$\mathrm{pH} 8,4$} & \multicolumn{2}{|c|}{$\mathrm{pH} \mathrm{7,0}$} \\
\hline & & $\mathrm{Com} \mathrm{Ca}^{2+}$ & Sem $\mathrm{Ca}^{2+}$ \\
\hline $\mid A C-8 \ldots \ldots \ldots \ldots$ & 0,300 & 0,292 & 0,284 \\
\hline$-L_{2} \ldots \ldots \ldots \ldots \ldots$ & 0,252 & 0,108 & 0,108 \\
\hline $\mid A C-8 X-L_{2} \ldots \ldots \ldots \ldots$ & 0,268 & 0,143 & 0,137 \\
\hline $\mid A C-8 \times-L_{2} \ldots \ldots \ldots \ldots \ldots$ & 0,244 & 0,125 & 0,108 \\
\hline $\mid A C-8 \times-L_{2} \ldots \ldots \ldots \ldots$ & 0,167 & 0,086 & 0,092 \\
\hline IAC-8 $\times-L_{2} \ldots \ldots \ldots \ldots$ & 0,222 & 0,086 & 0,086 \\
\hline$-L_{3} \ldots \ldots \ldots \ldots \ldots$ & 0,256 & 0,284 & 0,237 \\
\hline $\mid A C-8 x-L_{3} \ldots \ldots \ldots \ldots$ & 0,284 & 0,310 & 0,237 \\
\hline $\mid A C-8 \times-L_{3} \ldots \ldots \ldots \ldots$ & 0,194 & 0,284 & 0,208 \\
\hline $\mid A C-8 \times-L_{3} \ldots \ldots \ldots \ldots$ & 0,237 & 0,301 & 0,260 \\
\hline IAC-8 $x-L_{3} \ldots \ldots \ldots$ & 0,268 & 0,310 & 0,260 \\
\hline $\mid A C-8 \times-L_{3} \ldots \ldots \ldots \ldots$ & 0,174 & 0,310 & 0,260 \\
\hline $\mid A C-8 \times-L_{3} \ldots \ldots \ldots \ldots$ & 0,222 & 0,284 & 0,229 \\
\hline$-L_{1} \ldots \ldots \ldots \ldots \ldots$ & 0,032 & 0,187 & 0,174 \\
\hline
\end{tabular}

Segundo $\mathrm{KOCH}$ (1968), o ín $\mathrm{Ca}^{2+}$ estimula a lipoxigenase. CHRISTOPHER et al. (1972), porém, relatam que o ion $\mathrm{Ca}^{2+}$ estimula a atividade da lipoxigenase $L_{2}$ a $\mathrm{pH} 7,0$ e inibe a da $L_{3}$ em condições similares. 
Como ocorreu o estímulo do íon $\mathrm{Ca}^{2+}$ na atividade da lipoxigenase $\mathrm{L}_{2}$, a atividade do mutanie menos $\mathrm{L}_{3}$ é menor em pH 7,0 sem $\mathrm{Ca}^{2+}$ do que com $\mathrm{Ca}^{2+}$. Como a atividade da lipoxigenase $L_{2}$ é maior no $\mathrm{pH} 7,0$, o mutante menos $L_{2}$ tem menor atividade no $\mathrm{pH} 7,0$ em relação ao 8,4.

O perfil das isoenzimas por SDS-PAGE foi de difícil identificação, tendo-se preferido o método de focalização isoelétrica para a identificação dos mutantes, como se vê na figura 2, que apresenta as bandas isoelétricas do cultivar IAC8 e das linhagens Tohoko $n^{\circ} 74, \mathrm{PI} 86023$ e PI 408251: metade do gel (segmento I) foi colorido para proteína (RIGHETTI \& DRYSDALE, 1974) e metade (segmento Ii) para lipoxigenase (VERHUE \& FRANCKE, 1972): a mutante menos $L_{3}$ apresentou fracas as bandas $A, B$ e $C$; a mutante menos $L_{2}$, a banda $E$ forte no lugar da $D$, e a mutante menos $L_{1}$, fracas as bandas $A, D$ e .

De acordo com os resultados do presente trabalho, as análises quantitativas da atividade de lipoxigenase forneceram uma indicação do tipo de cultivar, facilitando a identificação final do mutante por focalização isoelétrica, uma vez que este último método necessita ser realizado só nas amostras com identificação quantitativa positiva.

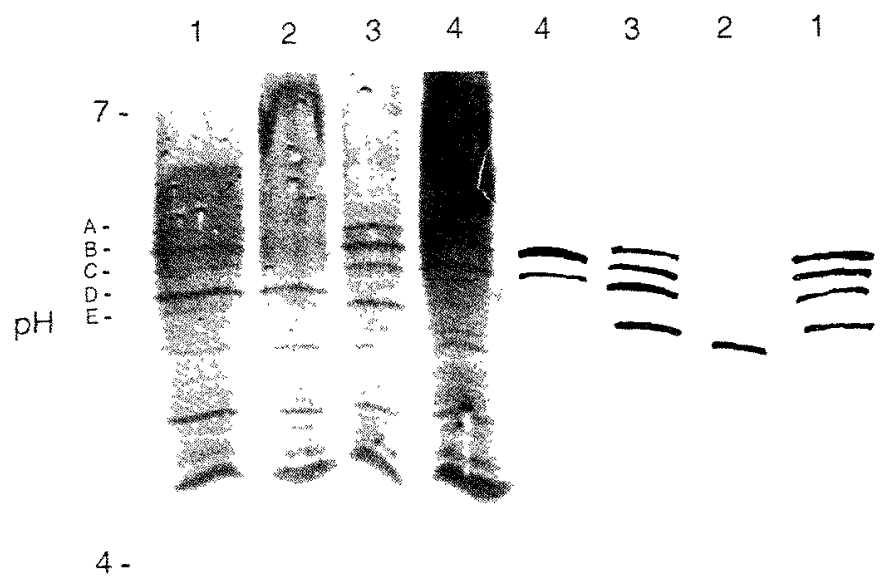

FIGURA 2. Resolução das isoenzimas de lipoxigenase de extratos de sementes de soja por focalização isoelétrica: 1: ‘IAC-8’; 2: Tohoko no 74; 3: PI 86023; 4: PI 408251. I: Coloração protéica. II: Coloração enzimática. 


\title{
SUMMARY
}

\section{LIPOXYGENASE ACTIVITY IN SOYBEAN LINES}

\begin{abstract}
Seed of soybeans, Glycine max L. (Merrill), cultivar IAC-8 and lines $A-5$, low linolenic acid content, $P \mid$ 408251, lacking $L_{1}$ mutant, $P I 86023$, lacking $L_{2}$ mutant, Tohoko $n^{\circ} 74$, lacking $L_{3}$ mutant, and $F_{2}$ population from the crosses between $\mid A C-8$ and mutant lines (lacking $L_{1}, L_{2}$ and $L_{3}$ ) were analysed for linolenic acid content, lipoxygenase activity and identification of mutant lines lacking $L_{1}, L_{2}$ or $L_{3}$ isoenzymes. The purpose was to evaluate methods to identify mutants lines of soybean for lipoxygenase and to verify a possible correlation between enzimatic activity and linolenic acid content. The enzimatic activity was estimated quantitatively by a colorimetric method and lipoxygenase isoenzymes detection by isoelectric focusing. The oil seed content was determined by gravimetry and the fatty acids content by gas liquid chromatography of their methyl esters. The lipoxygenase activity data was good to indicate mutant lines for the final identification using isoelectric focusing. It permits the use of this method for choosing lines in breeding program to obtain a good soybean oil stability. It was not observed correlation, in the material studied, between enzymatic activity and linolenic acid content.
\end{abstract}

Index terms: soybeans, Glycine max (L.) (Merrill), lipoxygenase, linolenic acid.

\section{REFERÊNCIAS BIBLIOGRÁFICAS}

ALLEN, J.C. Soybean lipoxygenase. 1. Purification, and the effect of organic solvents upon the kinetics of the reaction. European Journal Biochemistry, 4:201-208, 1968.

CHIBA, H.; TAKAHASHI, N. \& SASAKI, R. Enzymatic improvement of food flavor. II. Removal of beany flavor from soybean products by aldehyde dehydrogenase. Agricultural and Biological Chemistry, 43:1883-1889, 1979.

CHRISTOPHER, J.; PISTORIUS, E. \& AXELROB, B. Isolation of an isozyme of soybean lipoxygenase. Biochimica et Biophysica Acta, 198:12-19, 1970.

- PISTORIUS, E.K. \& BERNARD, A. Isolation of a third isoenzyme of soybean lipoxygenase. Biochimica et Biophysica Acta, 284:54-62, 1972.

DAVIES, C.S. \& NIELSEN, N.C. Genetic analysis of a null-allele for lipoxygenase-2 in soybean. Crop Science, 26:460-463, 1986.

DILLARD, M.G.; HENICK, A.S. \& KOCH, R.B. Differences in reactivity of legume lipoxidases. The Journal of Biological Chemistry, 236:37-40, 1961.

FUNK, M.O.; WHITNEY, M.A.; HAUSKENECHT, E.C. \& O'BRIEN, E.M. Resolution of the isoenzymes of soybean lipoxygenase using isoelecric focusing and chromatofocusing. Analytical Biochemistry, 146:246-251, 1985.

GUSS, P.L.; RICHARDSON, T. \& STAHMANN, M.A. The oxidation-reduction enzymes of wheat. III. Isoenzymes of lipoxidase in wheat fractions and soybean. Cereal Chemistry, 44:607-610, 1967. 
GUSS, P.L.; RICHARDSON, T. \& STAHMANN, M.A. Oxidation of various lipid substrates with unfractionated soybean and wheat lipoxidase. Journal of the American Oil Chemists Society, 45:272-276, 1968.

HAMMOND, E.G. \& FEHR, W.R. Registration of A5 germplast line of soybean. Crop Science, 23:192, 1983.

HARTMAN, L. \& LAGO, R.C.A. Rapid preparation of fatty acid methyl esters from lipids. Laboratory Practice, 22:475-477, 1973.

HILDEBRAND, D.F. \& HYMOWITZ, T. Inheritance of lipoxygenase-1 activity in soybean seeds. Crop Science, 22:851-853, 1982.

KITAMURA, K. Biochemical characterization of lipoxygenase lacking mutants, L-1-less, L-2-less, and L-3-less soybean. Agricultural and Biological Chemistry, 48:2339-2346, 1984.

; DAVIES, C.S.; KAIZUMA, N. \& NIELSEN, N.C. Genetic analysis of a null-allele for lipoxygenase-3 in soybean seeds. Crop Science, 23:924-927, 1983.

$\mathrm{KOCH}$, R.B. Calcium ion activation of lipoxidase. Archives of Biochemistry and Biophysics, 125:303-307, 1968.

; STERN, B. \& FERRARI, C.G. Linoleic acid and trilinolein as substrates for soybean lipoxidase (s). Archives of Biochemistry and Biophysics, 78:165-179, 1958.

LOWRY, O.H.; ROSEBROUGH, N.J.; FARR, A.C. \& RANDALL, R.J. Protein measurement with the Folin-phenol reagent. Journal of Biological Chemistry, 193:265-275, 1951.

MATTICK, L.R. \& HAND, D.B. Identification of a volatile component in soybean that contributes to the raw bean flavor. Journal of Agricultural and Food Chemistry, 17:15-17, 1969.

MUSTAKAS, G.C.; ALBRECHT, W.J.; McGHEE, J.E.; BLACK, L.T.; BOOKWALTER, G.N. \& GRIFFIN JUNIOR, E.L. Lipoxidase deactivation to improve stability, odor and flavor of full-fat soy flours. Journal of the American Oil Chemist's Society, 46:623-626, 1969.

RIGHETTI, P.G. \& DRYSDALE, J.W. Isoelectric focusing in gels. Journal of Chromatography, 98:271-321, 1974.

SESSA, D.J.; HONIG, D.H. \& RACKIS, J.J. Lipid oxidation in full-fat and defatted soybean flakes as related to soybean flavor. Cereal Chemistry, 46:675-686, 1969.

TRIEBOLD, H.O. \& AURAND, L.N. Food composition and analysis. New York, Van Nostrand, 1963. 497p.

VERHUE, W.M. \& FRANCKE, A. The heterogeneity of soybean lipoxygenase. Biochimica et Biophysica Acta, 284:43-53, 1972. 